\title{
Autocrine loop for IGF-I receptor signaling in SLUG-mediated epithelial-mesenchymal transition
}

\author{
RAMADOSS SIVAKUMAR ${ }^{1}$, HIRONORI KOGA ${ }^{1,2}$, KARUPPAIYAH SELVENDIRAN $^{1}$, \\ MICHIKO MAEYAMA ${ }^{1,2}$, TAKATO UENO $^{1}$ and MICHIO SATA ${ }^{1,2}$ \\ ${ }^{1}$ Research Center for Innovative Cancer Therapy, and Center of the 21st Century Center of Excellence Program for \\ Medical Science, ${ }^{2}$ Division of Gastroenterology, Department of Medicine, Kurume University, Kurume, Japan
}

Received August 12, 2008; Accepted October 6, 2008

DOI: 10.3892/ijo_00000155

\begin{abstract}
Akt, a downstream effector kinase of insulin receptor and insulin-like growth factor-I receptor (IGF-IR), is critically involved in epithelial-mesenchymal transition (EMT). The aim of this study was to assess the impact of SLUG in the IGF/IGF-IR/Akt axis. The SLUG-overexpressing MDCK (SLUG-MDCK) cell clones were used as the EMT model. In contrast to the parental cells and mock-transfected MDCK cells, SLUG-MDCK cells were markedly sensitive to IGFs, showing a clear tyrosine-phosphorylation in IGF-IR under serum-starved conditions. The IGF-IR of hepatocytes was highly activated by culture supernatants from SLUG-MDCK cells. This activation was inhibited by IGF-binding protein (IGFBP)-3, and by the IGF-IR inhibitor PQ401, leading to inactivation of Akt. This finding suggested establishment of autocrine IGF-IR signaling in the SLUG-MDCK cells. It is known that cells overexpressing receptor tyrosine kinases have an increased activity in Src kinase, which constitutively phosphorylates IGF-IR. In the present study, we found an increased phosphorylation of Src in SLUG-MDCK cells, and not in mock-MDCK cells; however, this Src activation was not always coupled with the constitutive activation of IGF-IR, since the specific Src inhibitor PP2 failed to decrease the IGF-IR phosphorylation. PP2 just attenuated the phosphorylation in Akt, not through IGF-IR inactivation, leading to apoptosis in SLUG-MDCK cells. Of interest, the inactivation of Akt by IGFBP-3 was dramatically enhanced in combination with the use of $\mathrm{PP} 2$, resulting in a significant apoptosis in SLUG-MDCK cells. These findings suggested that dual
\end{abstract}

Correspondence to: Dr Hironori Koga, Division of Gastroenterology, Department of Medicine, Liver Cancer Division, Research Center for Innovative Cancer Therapy, and Center for the 21st Century COE Program for Medical Science, Kurume University, Kurume 830-0011, Japan

E-mail: hirokoga@med.kurume-u.ac.jp

Key words: EMT, Akt, Snail, RTK, invasion targeting for IGF-IR and Src might be a potential therapeutic strategy in EMT-driven aggressive cancers.

\section{Introduction}

Epithelial-mesenchymal transition (EMT) is a process which confers migratory phenotype to polarized epithelial cells during embryogenesis. Recent evidence has suggested that a process similar to EMT occurred during the progression of epithelial tumors, endowing cancer cells with increased motility and invasiveness (1). Snail family transcriptional factors including SLUG were found to play key roles in inducing EMT mainly by suppressing the expression of E-cadherin (2-4).

Increased expression and activation of receptor tyrosine kinases (RTKs) are often linked to aggressive phenotypes in cancer (5). Activation of RTKs has recently been shown to induce expression of the Snail group of proteins leading to EMT (1), indicating an important role of the proteins in growth factor-mediated initiation of EMT. Insulin-like growth factor-I receptor (IGF-IR) is an important RTK involved in IGF-I and IGF-II-mediated mitogenic signaling and also linked to the development and progression of malignant cancers (6). IGF-IR activation has been shown to induce EMT by insulin receptor substrate (IRS)/phosphatidyl-inositol-3 kinase (PI3K)/Akt-mediated mechanisms (7-9). Breast cancer cells overexpressing IGF-IR have demonstrated depolarization and mesenchyme-like transition following IGF-I stimulation (10). Recently, Kim et al have clearly shown that constitutively active IGF-IR could confer in vivo tumorigenic growth to immortalized human mammary epithelial cells through upregulation of Snail (8), indicating an oncogenic role for IGF-IR. Indeed, many epidemiological studies from the late 1990s have suggested an insulin/IGF-I-cancer link $(11,12)$. Renehan et al confirmed the IGF-I-cancer link by a metaregression analysis of studies on major cancer sites, such as prostate, breast, colorectum, and lung (12). These clinical findings suggested that it could be worthwhile to investigate the IGF milieu in the SLUG-induced EMT caused in the well-polarized epithelial cells MDCK (Madin-Darby canine kidney), with a special focus on alterations in both cellular IGF production and receptor-mediated IGF signaling in those transformed cells.

Src, an intracellular tyrosine kinase, has been implicated as one of the key regulators of EMT, since overexpression 
or activation of Src altered the expression and intracellular localization of proteins including E-cadherin and B-catenin (13-15). Aggressive cancers overexpressing RTKs also show a high expression level of Src, suggesting that the membranous RTKs and the cytoplasmic tyrosine kinases may interact and synergistically promote the aggressiveness of these cancers (16). Indeed, IGF-IR and Src directly interact with each other through phosphorylation and participate together in cellular transformation and motility (17-19). Co-expression of these tyrosine kinases in human pancreatic cancer tissues has been reported (20), supporting partnership in mediating IGF-IR signaling.

We demonstrate here that SLUG augments the IGFstimulated phosphorylation of IGF-IR in an autocrine manner and simultaneously activates Src in the transformed MDCK cells. Because both the tyrosine kinases IGF-IR and Src participate in activation of Akt, a master regulator of cellular motility and anti-apoptosis, we further show that inhibition in both IGF-IR and Src leads to significant apoptosis in the mesenchymally-transformed cells.

\section{Materials and methods}

Materials. Recombinant human IGF-I, IGF-II, and IGF-binding protein (IGFBP)-3 were purchased from PeproTech (Rocky Hill, NJ). The Src kinase inhibitor PP2 and the PI3 kinase (PI3K) inhibitor LY294002 were purchased from Calbiochem (San Diego, CA). The IGF-IR inhibitor PQ401 was obtained from Tocris Bioscience (Ellisville, MO). Antibodies against SLUG, E-cadherin, vimentin, B-catenin, and phosphorylated (p-) IRS-1 (Tyr632) were obtained from Santa Cruz Biotechnology (Santa Cruz, CA). Antibodies for Snail, epidermal growth factor receptor (EGFR), p-EGFRs (Tyr992 and Tyr1068), IGF-IRß, p-IGF-IRß (Tyr1135/1136, rabbit monoclonal), p-PI3-kinase P85 (Tyr458), Akt, p-Akt (Ser473), phosphorylated Src (Tyr416), non-phosphorylated Src (Tyr416), poly-adenosine diphosphate ribose polymerase (PARP), and cleaved caspase- 3 and -7 were purchased from Cell Signaling Technology (Beverly, MA). Antibodies against actin and FLAG were from Sigma (Saint Louis, MO). The neutralizing antibody to IGF-IR (clone 3325) was obtained from R\&D Systems Inc. (Minneapolis, MN), and that to insulin receptor (IR) (clone 29B4) was obtained from Calbiochem. Enhanced chemiluminescence (ECL) reagents were obtained from Amersham Pharmacia Biotech (Buckinghamshire, UK), and the protein assay reagents were from Bio-Rad (Hercules, CA). All other reagents and compounds were analytical grades.

Cell lines and cultures. MDCK cells and Saos-2 cells obtained from RIKEN BioResource Center (Tsukuba, Japan) and the fetus-derived immortalized hepatocyte cell line OUMS-29 were used in this study. The OUMS-29 cell line has been well characterized to have cell polarity and several hepatocytespecific gene expressions, including albumin and asialoglycoprotein receptor $(21,22)$. Each cell line was grown in Dulbecco's modified Eagle's medium (Sigma-Aldrich Japan, Tokyo, Japan) supplemented with $10 \%$ heat-inactivated $\left(56^{\circ} \mathrm{C}, 30 \mathrm{~min}\right)$ fetal bovine serum (BioWest, Nuaill, France), $100 \mathrm{U} / \mathrm{ml}$ penicillin, and $100 \mu \mathrm{g} / \mathrm{ml}$ streptomycin (Invitrogen, Carlsbad, $\mathrm{CA}$ ) in a humidified atmosphere of $5 \% \mathrm{CO}_{2}$ at $37^{\circ} \mathrm{C}$.
Transfection of cDNAs and establishment of SLUG-overexpressing clones. The human SLUG clone (FLAG-tagged $S L U G$ in pPGS-CMV-CITE-Neo(-) vector) was a kind gift from Professor Eric Fearon (University of Michigan). The human Snail clone (accession no. NM_005985) was purchased from OriGene Technologies, Inc. (Rockville, MD). The human Twist clone was a kind gift from Professor Kimitoshi Kohno (University of Occupational and Environmental Health, Kitakyushu, Japan) (23). Transient transfection of the cDNAs was performed using TransIT ${ }^{\circledR}$-LT1 Transfection Reagent (Mirus Bio Corp., Madison, WI), according to the manufacturer's protocol. SLUG-FLAG-overexpressing clones (SLUG-MDCK) and mock-transfected clones (mockMDCK) were established as previously reported (24).

Immunoblot analysis. Immunoblotting was performed as previously reported (24). Samples containing cell lysate protein were separated on sodium dodecyl sulfate-polyacrylamide gel and then transferred onto equilibrated polyvinylidene difluoride membranes (Bio-Rad). After skimmed-milk blocking, the membranes were incubated with the primary antibodies. The bound antibodies were detected with horseradish peroxidase-labelled secondary antibodies using the enhanced chemiluminescence detection system (ECL Advanced kit). A positive signal from the target proteins was visualized using an image analyzer LAS-1000plus (Fujifilm, Tokyo, Japan), and the fold increase in the protein expressions was determined using an Image Gauge version 3.45 (Fujifilm).

IGF stimulation experiments. MDCK, mock-MDCK, SLUGMDCK, and OUMS-29 cells were plated in 100-mm dishes. Upon reaching 50-60\% confluency, the cells were starved in serum-free media for $36 \mathrm{~h}$ and then stimulated with IGF-I or IGF-II $(10 \mathrm{ng} / \mathrm{ml})$ for $30 \mathrm{~min}$. The cells were harvested and their lysates were subjected to immunoblot analysis. In transient transfection experiments, the transfected cells were cultured for $24 \mathrm{~h}$ in the serum-containing media with subsequent culture in serum-free media for $12 \mathrm{~h}$, and then exposed to IGF-II for $30 \mathrm{~min}$.

Experiments using IGFBP-3, PQ401, and culture supernatants. To investigate the local production and autocrine action of IGFs, $60 \%$-confluent MDCK, mock-MDCK, and SLUG-MDCK cells were serum-starved for $24 \mathrm{~h}$ or $35 \mathrm{~h}$, and then $100 \mathrm{ng} / \mathrm{ml}$ IGFBP-3 was added to the media and incubated for 12 or for $1 \mathrm{~h}$, respectively. When using the small molecule IGF-IR inhibitor PQ401, the cells were serum-starved for $35 \mathrm{~h}$ and exposed to the reagent $(10 \mu \mathrm{M})$ for $1 \mathrm{~h}$. After the exposure to IGFBP-3 or PQ401, the cells were harvested and used for immunoblotting. To further validate the establishment of the autocrine IGF/IGF-IR signaling loop in SLUG-MDCK cells, the culture supernatant was collected from the MDCK cells, the mock-MDCK cells, and from the SLUG-MDCK cells with 36-h serum starvation, and then these supernatants were used to examine the phosphorylation status in both the IGF-IR and the Akt in OUMS-29 cells.

Enzyme-linked immunosorbent assay to measure the concentrations of IGF-I. The concentration of IGF-I in the culture supernatants and in the cell lysates was quantified 
using the Quantikine ELISA kit (R\&D Systems) according to the manufacturer's protocol. In calculating the IGF-I concentrations in the culture supernatants, the basal value of the IGF-I concentration in the $10 \%$-serum-containing media was subtracted from the obtained raw data. All the ELISA experiments were performed using seven samples in duplicate.

Reverse transcription-polymerase chain reaction ( $R T-P C R)$. Total RNA was isolated from cells using the TRIzol reagent (Invitrogen Japan K.K., Tokyo, Japan), according to the manufacturer's instructions. RNA quantification was performed by spectrophotometry. The RT-PCR analysis was carried out using the GeneAmp PCR System 9700 (Applied Biosystems, Foster City, CA) under the following conditions: initial denaturation at $94^{\circ} \mathrm{C}$ for $4 \mathrm{~min}, 35$ cycles of amplification (denaturation at $94^{\circ} \mathrm{C}$ for $30 \mathrm{sec}$, annealing at $55^{\circ} \mathrm{C}$ for $30 \mathrm{sec}$, and extension at $72^{\circ} \mathrm{C}$ for $30 \mathrm{sec}$ ), and extension at $72^{\circ} \mathrm{C}$ for $10 \mathrm{~min}$. Reagents for RT-PCR were obtained form Takara Bio Inc. (Shiga, Japan). The sequences (5'-3') for the primer pairs of IGF-I and B-actin (an internal control), respectively, were as follows: for IGF-I, ATGTCCTCCTCGCATCTCTT (forward) and TCCCTCTACTTGCGTTCTTC (reverse); and for $\beta$-actin, TGGCACCACACCTTCTACAACGAG (forward) and AGAGGCATACAGGGACAGGACAGC (reverse). The PCR products (350 bp for IGF-I mRNA, and $180 \mathrm{bp}$ for $\beta$-actin) were electrophorased on $2 \%$ agarose gel, and stained with ethidium bromide.

RNA interference for SLUG. Three sets of small interfering RNAs (siRNAs) corresponding to nucleotide sequences of human SLUG (accession no. NM_003068) were synthesized and purchased from Invitrogen. The Stealth ${ }^{\mathrm{TM}}$ Select RNAi sequences for the primer sets were as the followings: no. 1, UGGAGUAACUCUCAUAGAGAUACGG (sense) and CCGUAUCUCUAUGAGAGUUACUCCA (antisense); no. 2, UAAUGUGUCCUUGAAGCAACCAGGG (sense) and CCCUGGUUGCUUCAAGGACACAUUA (antisense); and no. 3, AUCAGAAUGGGUCUGCAGAUGAGCC (sense) and GGCUCAUCUGCAGACCCAUUCUGAU (antisense). The negative control duplex was also obtained from Invitrogen. The siRNA duplexes at the final concentration of $10 \mathrm{nM}$ were transfected into Saos-2 cells, which were known to express SLUG (25), using the HiPerFect Transfection Reagent (Qiagen K.K.), according to the manufacturer's protocols. At $48 \mathrm{~h}$ after the siRNA transfection into Saos-2 cells, the cell lysates were subjected to immunoblot analysis to determine the most effective siRNA to inhibit the SLUG expression from the above-described three siRNAs. Using the most effective siRNA, the IGF-I concentration in the culture supernatants of the SLUG-down-regulated Saos-2 cells was compared with that of the control cells using ELISA.

Inactivation of Src kinase by PP2. MDCK, mock-MDCK, and SLUG-MDCK cells at $60 \%$-confluent state were serumstarved for 24 or $35 \mathrm{~h}$, and then exposed to $10 \mu \mathrm{M}$ PP2 for 12 or for $1 \mathrm{~h}$, respectively. The harvested cells were subjected to immunoblotting. To confirm the effects of PP2 even in the serum-containing media, the $60 \%$-confluent SLUG-MDCK cells were treated with PP2 for $12 \mathrm{~h}$, and used for the immunoblot analysis.
Combination of IGFBP-3 with PP2. The apoptosis-inducing potentials of IGFBP-3 and of PP2 were examined. MDCK, mock-MDCK, and SLUG-MDCK cells were cultured in serum-free media for $24 \mathrm{~h}$ up to the $60 \%$-confluent state. The cells were incubated with two different dosages of PP2, at 5 and $10 \mu \mathrm{M}$, for $12 \mathrm{~h}$. After the incubation with PP2, both the attached cells and the floating cells were collected, and their lysates were subjected to the immunoblot analysis to investigate the expression levels in apoptotic markers such as PARP, cleaved caspase- 3 and -7 . To assess the possible enhancement in inducing apoptosis in the combined use of IGFBP-3 with PP2, the 24-h serum-starved SLUG-MDCK cells were incubated with $100 \mathrm{ng} / \mathrm{ml} \mathrm{IGFBP-3}$ and $10 \mu \mathrm{M}$ PP2 for $12 \mathrm{~h}$. The immunoblot analysis was performed to detect the above-mentioned apoptotic markers.

Statistical analysis. Statistical significance was assessed using Mann-Whitney $\mathrm{U}$ test. $\mathrm{P}<0.05$ was considered statistically significant.

\section{Results}

Constitutive activation of Akt and of IGF-IR in SLUGinduced EMT. SLUG-MDCK cells showed a spindle-shaped appearance without forming any epithelial sheet-like pattern as did the parental MDCK cells (Fig. 1A). An undetectable expression level of E-cadherin was confirmed in the SLUGMDCK cells, accompanied by a decrease in the $\beta$-catenin expression level and a clear increase in the mesenchymal marker vimentin (Fig. 1B). In a survey of Akt and its related tyrosine kinases, we found remarkable phosphorylation in both the Akt and the Src in the SLUG-MDCK cells. Indeed, the $\mathrm{p}$-Akt/Akt ratio by densitometry from three independent experiments was 10.5-fold increased in SLUG-MDCK cells compared with that in MDCK cells or in mock-transfected cells (Fig. 1C). Then, we focused on IGF-IR, an upstream receptor of Akt, and found that the receptor was constitutively activated in these cells. The p-IGF-IRß/IGF-IRß ratio by the densitometry was 41.5-fold increased in SLUG-MDCK (Fig. 1D). IRS-1, a signal mediator recruited to $ß$-subunits of IGF-IR, was phosphorylated at tyrosine 632, suggesting activation in the IGF-IR signaling (Fig. 1D). Destruction of epithelial cellcell contact by SLUG markedly reduced the expression level of EGFR as well as that of its activated phosphorylated forms (Fig. 1E).

Enhanced sensitivity to IGFs by SLUG. To assess whether or not the increased p-IGF-IRß was functional in mediating IGF signaling, stimulation experiments were performed using IGF-I and IGF-II. In this experiment, the immortalized human hepatocyte cell line OUMS-29 was set as the IGFsensitive control. Indeed, as shown in Fig. 2, the IGF-IRß and the Akt of OUMS-29 cells were highly phosphorylated by IGF-I, and this was strongly inhibited by the neutralizing antibody to IGF-IRß, but not by the neutralizing antibody to IR. This suggested that Akt was activated mainly through the IGF-IRß phosphorylation, but not through the IR phosphorylation, which could also be detected by the anti-p-IGF-IRß (Tyr1135/1136) antibody used. After serum starvation for $36 \mathrm{~h}$, the MDCK cells, mock-MDCK cells, the SLUG-MDCK 
A

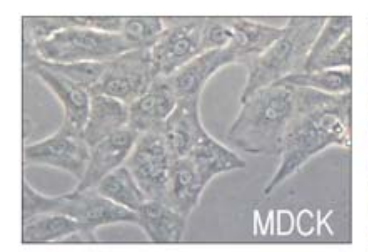

B

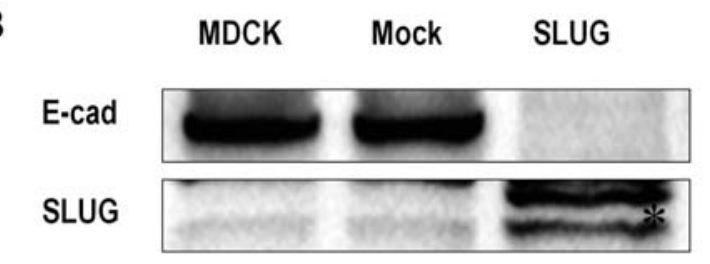

FLAG

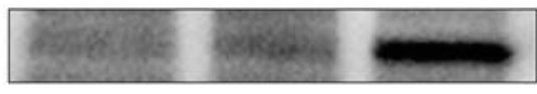

$\beta$-cat

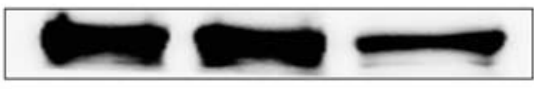

Vim

Actin
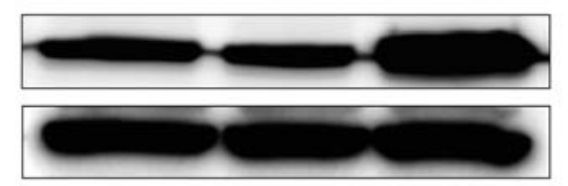

C

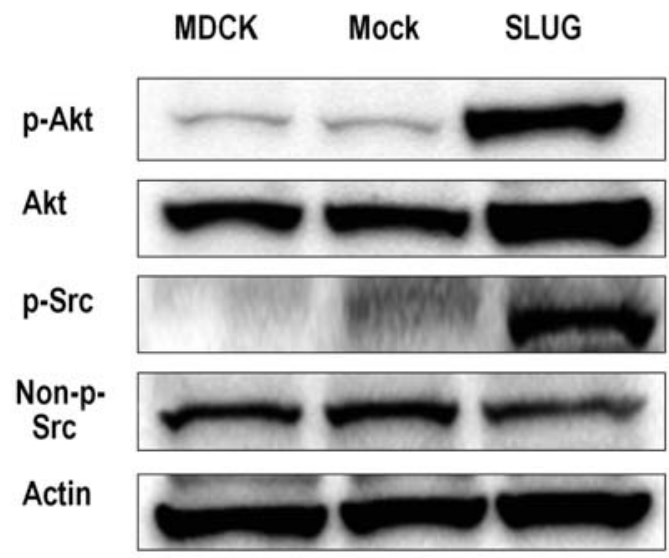

D

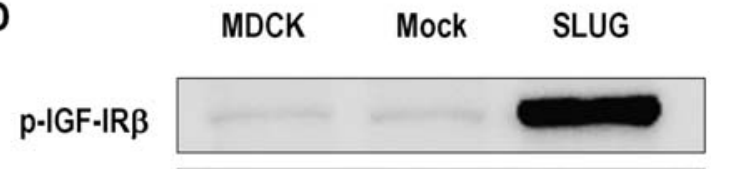

IGF-IR $\beta$

p-IRS-1

(Tyr-632)

Actin
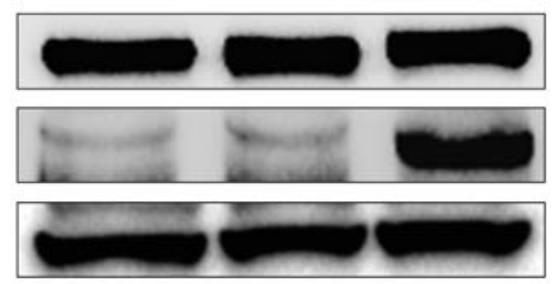

E

EGFR

p-EGFR

(Y992)

$\mathrm{p}$-EGFR

(Y1068)

Actin
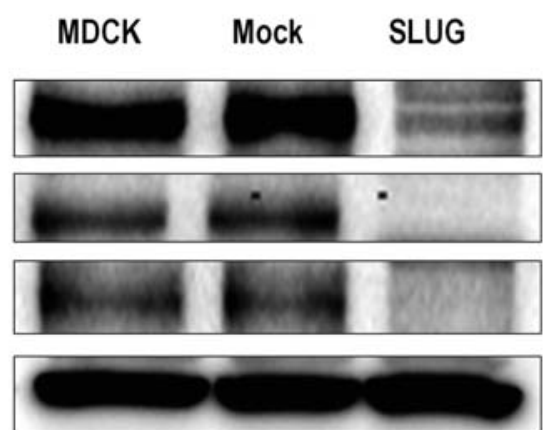

Figure 1. A, Phase-contrast microscopy demonstrates a fibroblastic appearance in SLUG-MDCK cells. B, Loss of the E-cadherin expression, a decrease in the ß-catenin (ß-cat) expression, and an increase in the vimentin (Vim) expression are demonstrated, confirming successful EMT in the SLUG-overexpressing clone. *Endogenous expression of SLUG. C, Expression levels in Akt, p-Akt, and $\mathrm{p}$-Src are markedly increased in SLUG-MDCK cells. D, Constitutively high levels in phosphorylation of both IGF-IRß and IRS-1 (Tyr632) are found in the SLUG-MDCK cells. E, Loss of expression and activation of EGFR is shown in SLUG-MDCK cells.

cells, and the OUMS-29 cells were stimulated with IGF-I or IGF-II. Phosphorylation in IGF-IRß, IRS-1, PI3K, and Akt was shown in each of the latter three IGF-stimulated cell lines; however, much greater activation was found in the SLUG-MDCK cells compared with the mock-MDCK cells (Fig. 3) (data from MDCK cells, not shown).

Autocrine IGF/IGF-IR loop in SLUG-induced EMT. To confirm the involvement of the IGF/IGF-IR axis in the constitutive activation of Akt, we used the IGF blocker IGFBP-3 and the IGF-IR inhibitor PQ401 to suppress stimuli from the ligands. Even under serum-starvation, profound expressions in both p-IGF-IRß and p-Akt were found in the SLUG-MDCK cells, raising the possibilities that the stimuli to the IGF-IR of the cells were derived from themselves. Indeed, the activation of IGF-IR was clearly suppressed by IGFBP-3 added into the culture media of the SLUG-MDCK cells, leading to a subsequent decrease in the phosphorylation of Akt (Fig. 4A). The similar inactivation of the IGF-IR in the SLUG-MDCK cells was confirmed using PQ401, leading to more profound Akt inactivation (Fig. 4B). However, the suppression of IGF-IR activity by the IGFBP-3 exposure was partially cancelled at $12 \mathrm{~h}$, suggesting that continuous production of IGFs from the SLUG-MDCK cells nearly neutralized the effect of IGFBP-3 (Fig. 4A). If the SLUGMDCK cells produced and secreted IGFs into the culture media, the supernatants should have some potential to activate IGF-IR and Akt in the IGF-sensitive hepatocytes OUMS-29 cells. Indeed, the supernatants from the SLUG-MDCK cells remarkably induced phosphorylation in both the IGF-IR and the Akt of the OUMS-29 cells. In contrast to the high potential of the SLUG-MDCK-derived supernatants, the supernatants from the mock-MDCK cells did not induce any clear phosphorylation in the two molecules in the OUMS-29 cells (Fig. 4C). These phosphorylations caused by the SLUGMDCK-derived supernatants were inhibited by IGFBP-3, though partially (Fig. 4C), indicating that the supernatants contained ligands for IGF-IR. These findings suggested that the IGF/IGF-IR-driven Akt activation occurred in an autocrine manner in the SLUG-overexpressing cells. Indeed, an $~ 10$-fold 


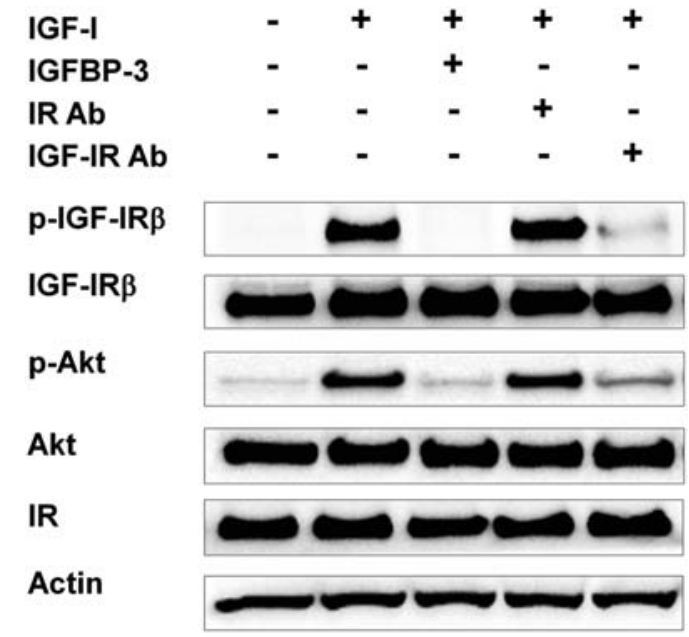

Figure 2. The hepatocye-derived OUMS-29 cells (OUMS) were cultured to $60 \%$-confluency, and then serum-starved for $36 \mathrm{~h}$. The cells were stimulated with IGF-I $(10 \mathrm{ng} / \mathrm{ml})$ with or without IGFBP-3 $(100 \mathrm{ng} / \mathrm{ml})$ or the neutralizing antibodies to insulin receptor (IR) (clone 29B4, $25 \mu \mathrm{g} / \mathrm{ml}$ ) and IGF-IR (clone $3325,10 \mu \mathrm{g} / \mathrm{ml}$ ) for $30 \mathrm{~min}$. After the incubation, the prepared cell lysates were used for the immunoblot analysis. increase in the concentration of IGF-I was found in the culture supernatants of the SLUG-MDCK cells compared with the IGF-I concentration in those of the mock-MDCK cells (Fig. 5A). Consistent with the trend obtained from the supernatant data, a significant increase in the concentration of IGF-I was also demonstrated in the cell lysates from the SLUG-MDCK cells (Fig. 5A). A greater expression level in the IGF-I mRNA was found in the SLUG-MDCK cells: the ratio IGF-I/ $/$-actin in the band intensity was 9.6-fold increased in the SLUG-MDCK cells (Fig. 5B). In the SLUG siRNA experiments with the IGF-I-producing Saos-2 cells, knockdown of the SLUG expression caused a significant decrease in the IGF-I production (Fig. 5C and D). These findings showed direct evidence of the augmented IGF production in a SLUG-dependent manner.

SLUG-activated Src contributed to Akt activation. Previous reports have suggested that overexpression of constitutively active Src in cells caused phosphorylation of IGF-IR and made the cells more sensitive to IGFs $(17,26)$. Because a marked activation (phosphorylation) of Src was found in our
A

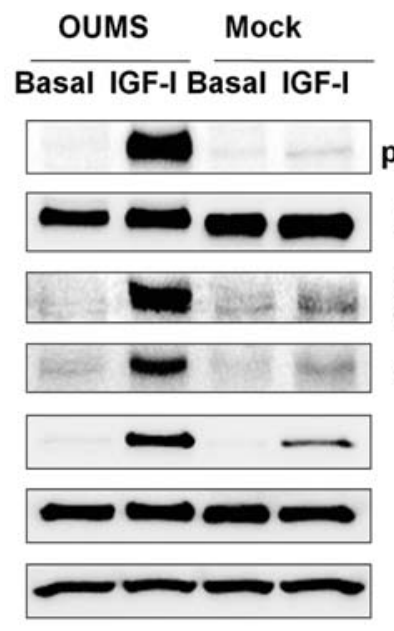

B $\frac{\text { OUMS }}{\text { Basal IGF-IIBasal IGF-II }}$

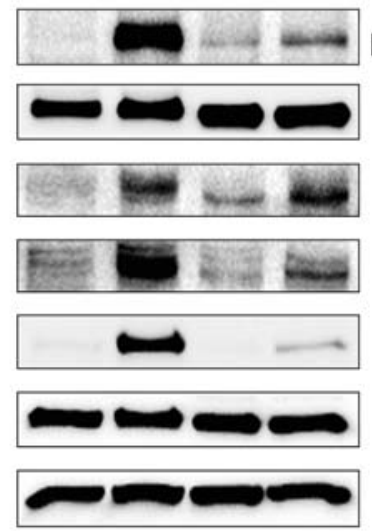

C
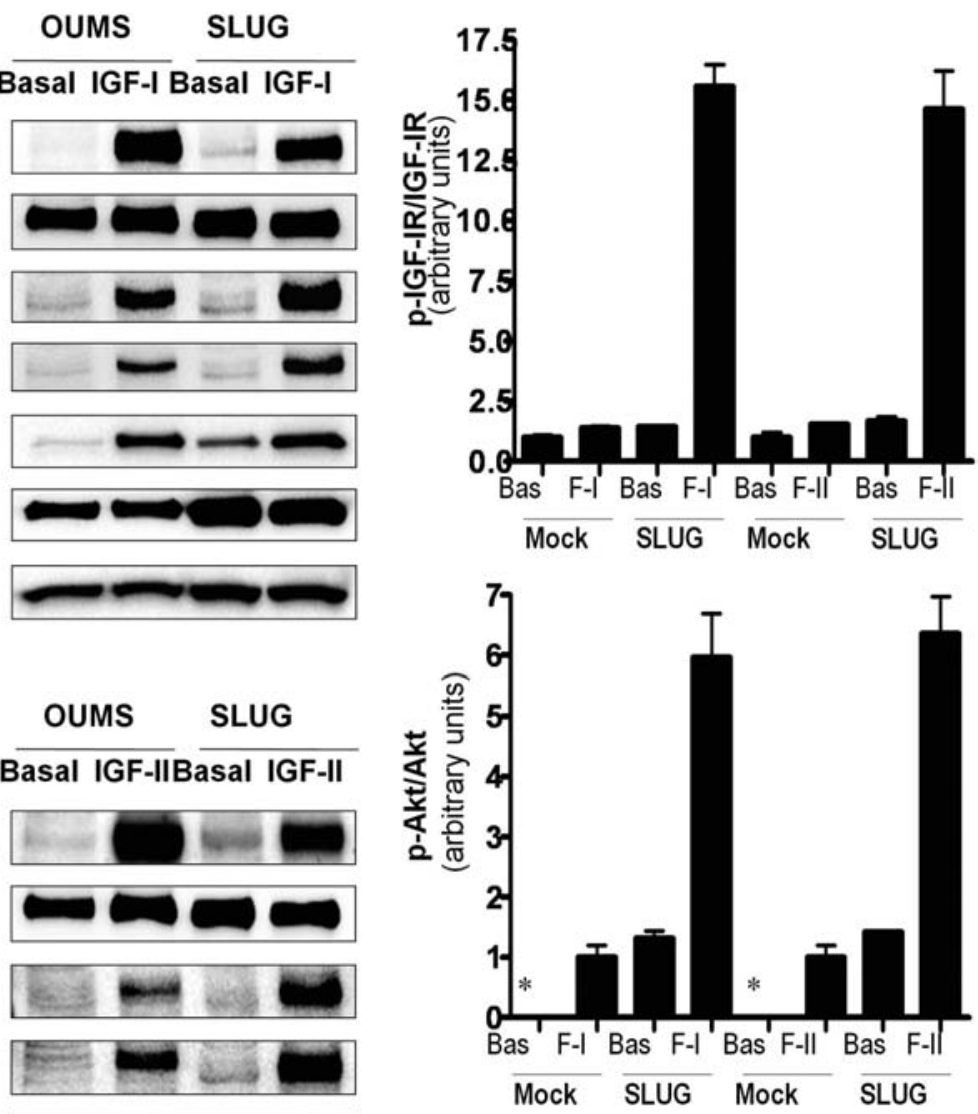

Figure 3. A and B, Phosphorylation in IGF-IRß, IRS-1, PI3K, and in Akt, is shown in the IGF-I (A)- and IGF-II (B)-stimulated cell lines (data from MDCK cells, not shown); however, much greater activation is found in the SLUG-MDCK cells (SLUG) than in the mock-MDCK cells (mock). OUMS-29 cells (OUMS) are the positive control for the IGF-sensitive cells. C, The densitometric analyses of visualized bands for p-IGF-IRß and p-Akt. The density ratios p-IGF-IRß/IGF-IR and p-Akt/Akt are shown as mean \pm SD of 3 independent experiments. *Not measured. Bas, basal. FI, IGF-I. FII, IGF-II. 
A

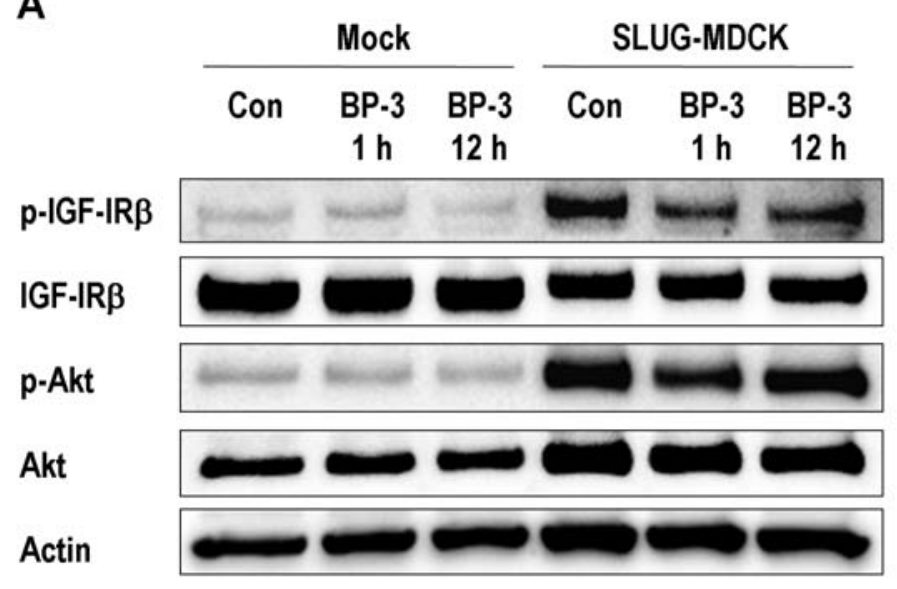

B

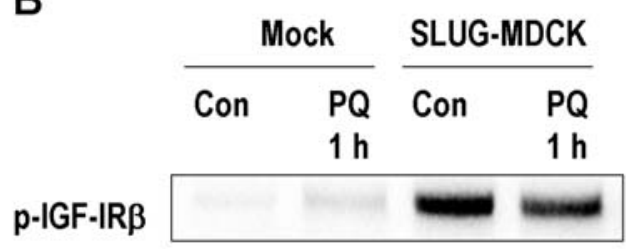

IGF-IR $\beta$

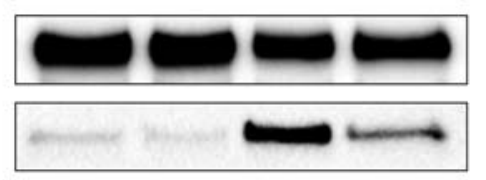

Akt

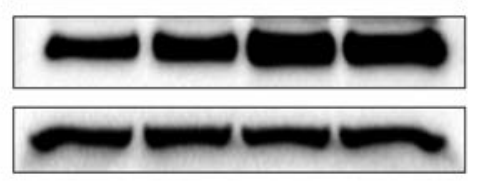

Actin

\section{p-Akt}

\section{IGF-II}

IGFBP-3

$\begin{array}{llllllll}\text { Mock-supernatant } & - & - & - & + & + & - & - \\ \begin{array}{l}\text { SLUG-MDCK. } \\ \text { supernatant }\end{array} & - & - & - & - & - & + & +\end{array}$

p-IGF-IRB

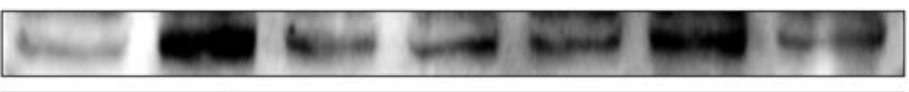

p-Akt

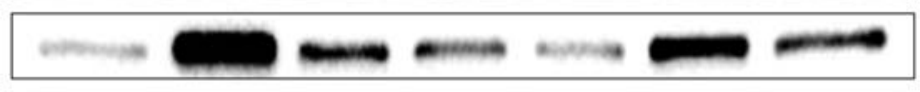

Actin

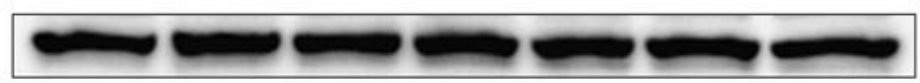

Figure 4. Immunoblot analysis representing the local production and the autocrine action of IGFs. A, After serum-starvation for 24 or 35 h, they were incubated with IGFBP-3 (BP-3) (100 ng/ml) for 12 or for $1 \mathrm{~h}$, respectively. Con, control. In B, PQ401 (PQ) (10 $\mu \mathrm{M})$ was added into the culture medium after serumstarvation for $35 \mathrm{~h}$, and then incubated for $1 \mathrm{~h}$. C, The mock-MDCK cells and the SLUG-MDCK cells were serum-starved for $36 \mathrm{~h}$. After the serum-starvation, the culture media were collected and centrifuged to remove floating cells and debris. About $60 \%$-confluent OUMS-29 cells were cultured in serum-free media for $24 \mathrm{~h}$, and then the medium was replaced with fresh serum-free medium containing IGF-II (10 ng/ml), the culture supernatants from mock-MDCK cells, or with the culture supernatants from SLUG-MDCK cells. To suppress the effect of the IGFs, the cells were incubated with $100 \mathrm{ng} / \mathrm{ml}$ IGFBP-3 for $30 \mathrm{~min}$. A-C, Data from MDCK cells, not shown.

study (Fig. 1C), we focused on the Src-IGF-IR link in the SLUG-induced EMT model using the specific Src inhibitor PP2. PP2 strongly inhibited the constitutive activation of Src in a serum-starved condition; however, this inactivation of Src did not affect the phosphorylation level of IGF-IR, while it did lead to inactivation of Akt (Fig. 6A) (data from MDCK cells, not shown). Similar findings were demonstrated in the SLUG-MDCK cells cultured in serum-containing media (Fig. 6B). These findings indicated that Src did not regulate the phosphorylation of Akt thorough the IGF-IR pathway, at least not in the EMT model used (Fig. 6A). Of interest, in concert with the down-regulation in p-Akt by the PP2 treatment, clear apoptosis was found in the SLUG-MDCK cells and not in the mock-MDCK cells, showing cleavage in PARP (Fig. 6A). This implied that targeting Src was effective on inducing apoptosis in mesenchymally-transformed cells, although this single targeting was insufficient to completely inactivate Akt.

Dual targeting for IGF-IR and Src efficiently induced apoptosis in EMT. Clear apoptosis was shown in the serumstarved SLUG-MDCK cells in a PP2-dosage-dependent manner, involving caspase activation including caspase- 3 and caspase-7 (Fig. 6C). In contrast, there was no clear increase in the expression level of the cleaved caspases in the serumstarved mock-MDCK cells (data from MDCK cells, not shown). To establish a more effective way to induce apoptosis in the transformed cells, we conducted combined exposure of IGFBP-3 with PP2 to reduce the Akt activity, which was responsible for anti-apoptosis. Under the serum-starved conditions, $100 \mathrm{ng} / \mathrm{ml}$ IGFBP-3 and $10 \mu \mathrm{M}$ PP2 demonstrated a significant decrease in the expression level of p-Akt, leading to a marked enhancement in apoptosis (Fig. 6D). Treatment with IGFBP-3 did not show any detectable apoptosis in these conditions.

Snail also enhanced IGF-IR signaling. The above results obtained using the stably-transformed SLUG-MDCK cells showed activation of both IGF-IR and Src; however, this system did not always mimic the behavior of cancer cells in vivo. The transformed cancer cells often reverse their phenotypes to those at the epithelial side with timely switching in the transcription factors (27). Thus, we performed transient gene expression experiments in the MDCK cells using Snail 


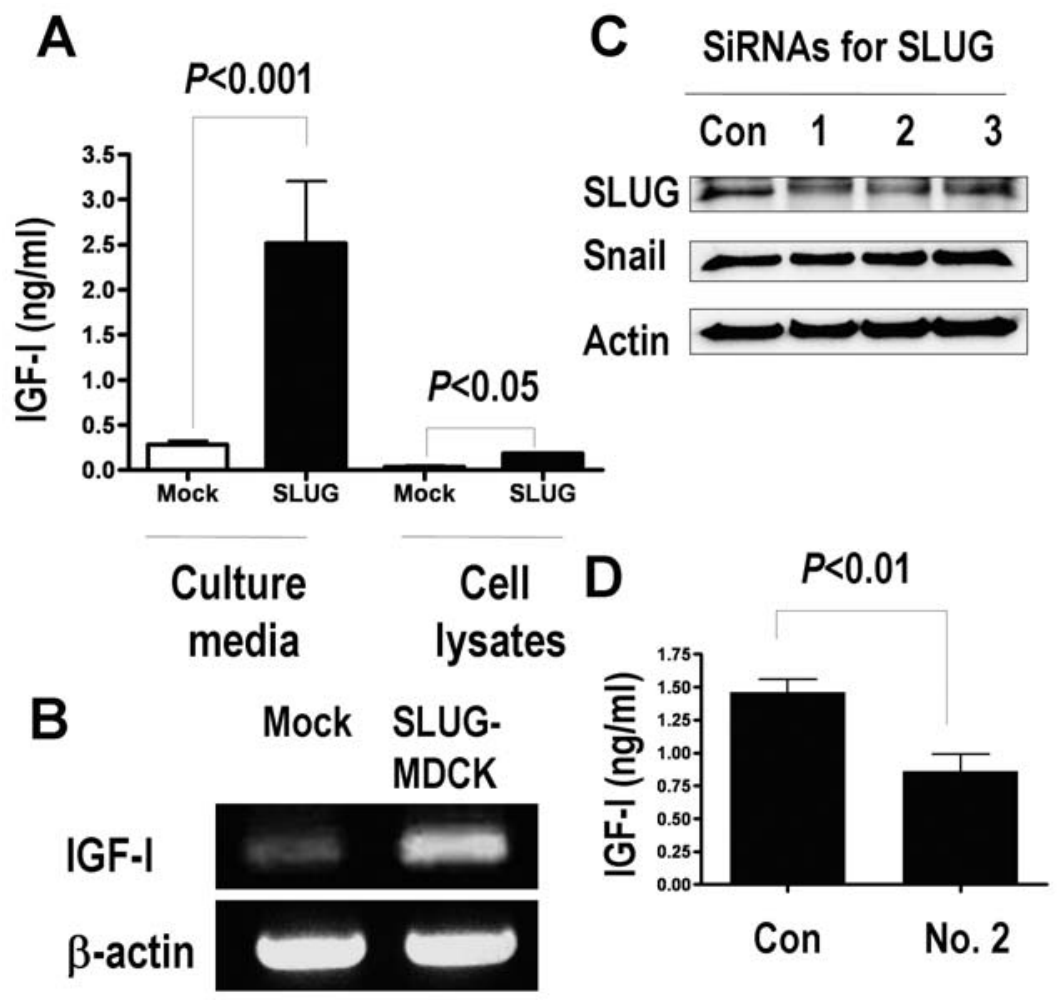

Figure 5. A, The IGF-I ELISA. MDCK, mock-MDCK cells (mock), and SLUG-MDCK cells (SLUG) were cultured in $10 \%$-serum-containing media for $36 \mathrm{~h}$ up to $80 \%$-confluency, then the culture supernatants were used for IGF-I quantification. The basal concentration of IGF-I in the $10 \%$-serum-containing medium was $0.16 \mathrm{ng} / \mathrm{ml}$. This value was subtracted from each of all the IGF-I data prior to statistical analysis. The cell lysates were prepared from the cell pellets after removing the culture supernatants, and the protein level in the lysates was adjusted to $7 \mathrm{mg} / \mathrm{ml}$. Data from MDCK cells, not shown. B, IGF-I mRNA levels by RT-PCR. Data from MDCK cells, not shown. C, The differential efficacy to knock-down the SLUG expression in Saos-2 cells using the three synthesized siRNAs. Con, negative control duplex RNA. D, The IGF-I ELISA for the culture supernatants of the control-siRNA-transfected Saos-2 cells and of the No. 2-siRNA-transfected Saos-2 cells.

and SLUG to assess the activation of IGF-IR and Src (Fig. 7). The transient expression of Snail clearly decreased the expression level of E-cadherin, and increased the expression levels of p-IGF-IRß and p-Src in a Snail-cDNA-dosagedependent manner, leading to a profound increased expression of p-Akt (Fig. 7A). Also in this transient gene expression system, the hypersensitivity to IGF was confirmed in both SLUG and Snail transfectants (Fig. 7B). However, another EMT-regulating transcription factor Twist did not show any detectable alteration in the expression level of p-IGF-IRß or p-Akt (Fig. 7C).

\section{Discussion}

In the present study, we have demonstrated the following new findings in the SLUG-induced EMT model: i) the constitutive activation of IGF-IR, ii) the increased sensitivity to IGFs through IGF-IR occurred in an autocrine manner, iii) the non-membranous type of tyrosine kinase Src contributed to the Akt activation without phosphorylating IGF-IR, and iv) targeted inhibition of both IGF-IR and Src resulted in a marked enhancement in apoptosis of the transformed cells.

It is well known that Akt activity is induced by ligand stimulation of growth factor receptors, such as IGF-IR and the EGF family of receptors (8), leading to pleiotropic effects on normal and cancerous cells (28). In the EMT model used in this study, however, the responsible RTK for the Akt activation was IGF-IR, and not EGFR which was absent in the transformed cells. Although the mechanism of this striking RTK switching was unclear, the switching demonstrated the potential to alter the cellular sensitivity to the ligands such as IGFs and EGF in a certain type of cell. In a previous study using human keratinocytes, findings suggested distinct roles for the two RTKs, showing that IGF-I promoted cell migration in an EGF-independent manner, since antibody to the EGFR failed to block IGF-I-enhanced cell migration (29). In a clinical setting, alteration in the RTK profile in cancer cells may impact on the therapeutic efficacy of emerging RTK-targeting drugs. Indeed, it has recently been shown that cancer cells having undergone EMT losing the expression of p-EGFR were insensitive to erlotinib, an EGFR inhibitor (30). Evidence including our findings of the EMT-associated RTK switching might provide insights into understanding and even overcoming the drug resistance of cancer cells.

Establishment of the EMT-driven autocrine signaling loops has recently been reported, including the platelet-derived growth factor (PDGF)/PDGFR signaling and the transforming growth factor- $\beta$ (TGF- $\beta$ )/TGF- $\beta$ R signaling $(31,32)$. In the present study, the IGFs/IGF-IR signaling was first identified as another autocrine system in EMT. The production and secretion of IGFs from the SLUG-MDCK cells was, at least in part, attributable to the constitutive stimulation of Akt via IGF-IR even under long-term serum-free condition. This autocrine IGF-IR signaling might be maintained by a possible 
A

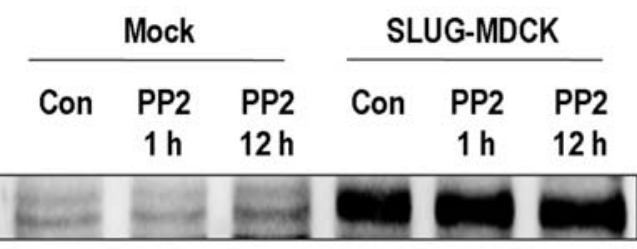

IGF-IR $\beta$

p-Akt

Akt

p-Src

Non-p-Src

PARP

Actin
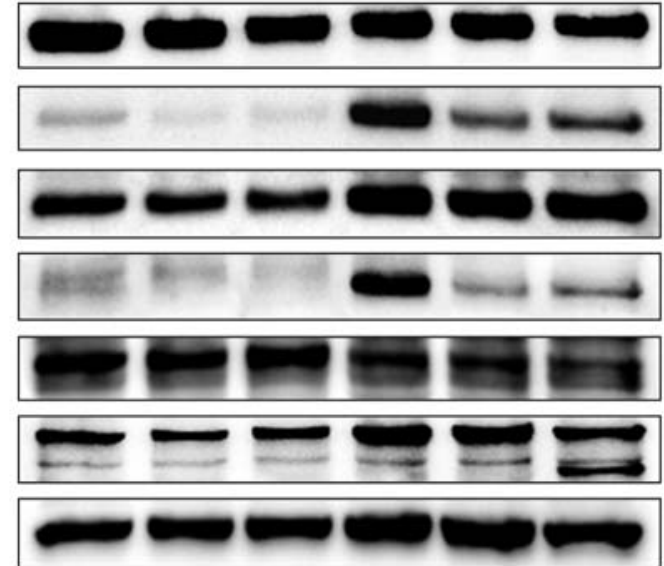

B

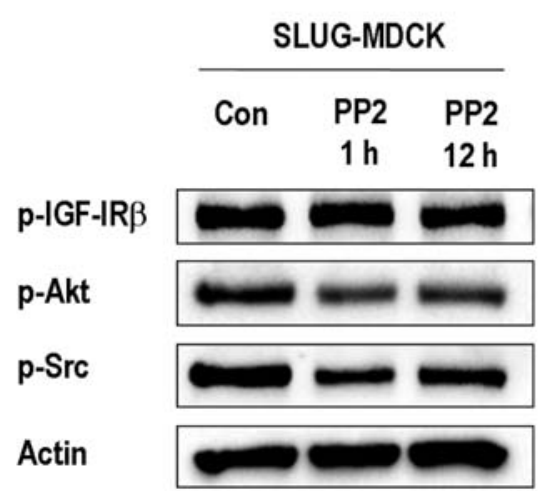

C

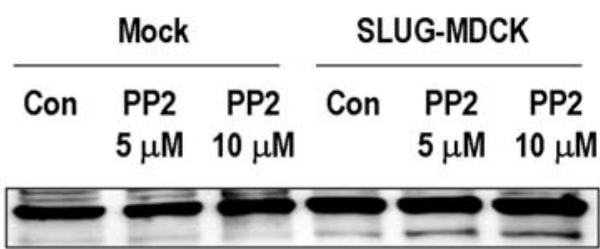

Cl. Cas-3

Cl. Cas-7

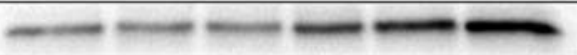

Actin

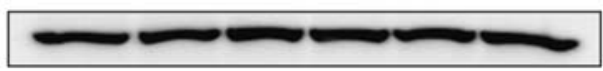

D

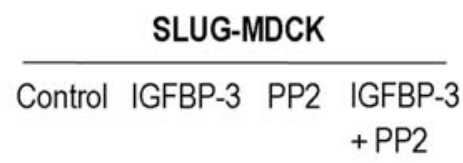

p-Akt

PARP
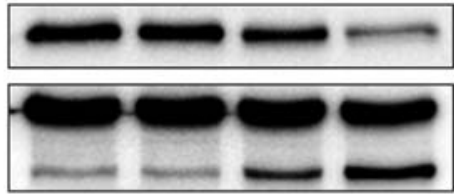

Cl. Cas-3

Cl. Cas-7

Actin

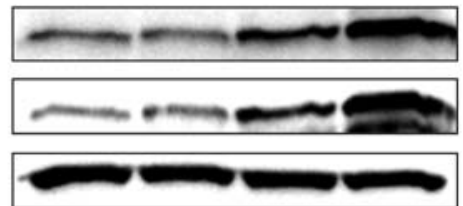

Figure 6. A, Activation of Src kinases is not coupled with constitutive activation of IGF-IR in SLUG-MDCK cells under serum-starved condition. PP2 inhibits Src in the SLUG-MDCK cells, leading to a marked decrease in the expression level of p-Akt. Although the p-Src and p-Akt expression levels are slightly recovered at $12 \mathrm{~h}$ of the PP2 treatment, clear apoptosis is found, showing a cleaved form of PARP in the SLUG-MDCK cells. The expression level of p-IGF-IRß is independent of the PP2-induced Src inactivation. Data from MDCK cells, not shown. B, Immunoblot analysis in the SLUG-MDCK cells cultured in serum-containing medium. PP2 exposure also shows the decreased expression of p-Akt. C, Immunoblot analysis demonstrating the dosagedependent apoptosis-inducing potential of PP2 in the SLUG-MDCK cells. 60\%-confluent MDCK cells, mock-MDCK cells, and SLUG-MDCK cells were cultured in serum-free media for $24 \mathrm{~h}$ and then incubated with 5 or $10 \mu \mathrm{M}$ PP2 for $12 \mathrm{~h}$. Data from MDCK cells, not shown. D, Immunoblot analysis shows enhanced apoptosis of the SLUG-MDCK cells by simultaneous blocking of the IGF-IR signaling and the Src kinase activity using IGFBP-3 and PP2, respectively.

feed forward mechanism in the transcription of SLUG (33). The autocrine loop was not completely inhibited by IGFBP-3, suggesting that another mechanism was involved in the Akt activation. Src is known to participate in Akt activation in aggressive cancers co-expressing highly activated IGF-IR $(17,19)$, phosphorylating IGF-IR $(17)$. Thus, we focused on the expression level of the activated $\mathrm{Src}(\mathrm{p}-\mathrm{Src})$ and its possible Akt-activating role in SLUG-MDCK cells, and found that Src was constitutively phosphorylated even in the serum-starved SLUG-MDCK cells and positively contributed to the Akt activation. Indeed, the inhibition of Src activity by PP2 clearly resulted in the inactivation of Akt; however, this inhibition did not always link to the inactivation of IGF-IR. These findings suggested that Src served as an upstream activator of Akt, independent of its IGF-IR-phosphorylating action in the SLUG-induced EMT. In the present study, it still remained unclear how Src was activated through the SLUG induction. Recently, it has been demonstrated that Src interacted with signal transducer and activator of transcription 3 (STAT3) (34), a transcriptional regulator of EMT, raising possibility for the direct interaction between EMT-regulating transcription factors, including SLUG, and Src.

Of interest, the expression level of the endogenous SLUG was increased in SLUG-MDCK clones, suggesting a possible autocrine loop in the transcriptional mechanism of SLUG. This finding may imply that exogenous EMT-causing stimuli can alter the endogenous expression system in SLUG, allowing participation of endogenous SLUG in maintaining mesenchymal transformation. Regarding the EMT-regulating transcription factors used in this study, the basic helixloop-helix-type Twist did not show any enhancing effects on phosphorylation of IGF-IR, IRS-1, or Akt, in contrast to the 


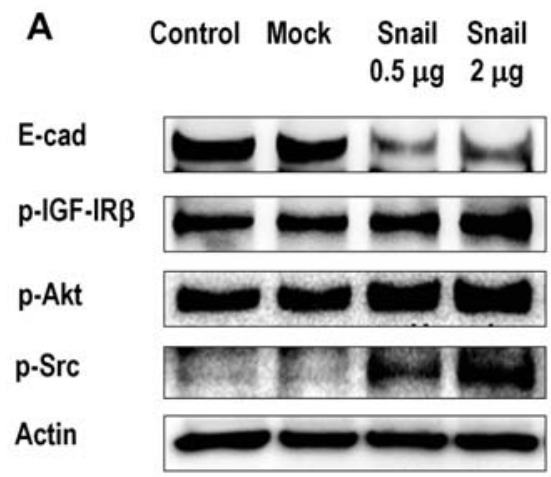

B

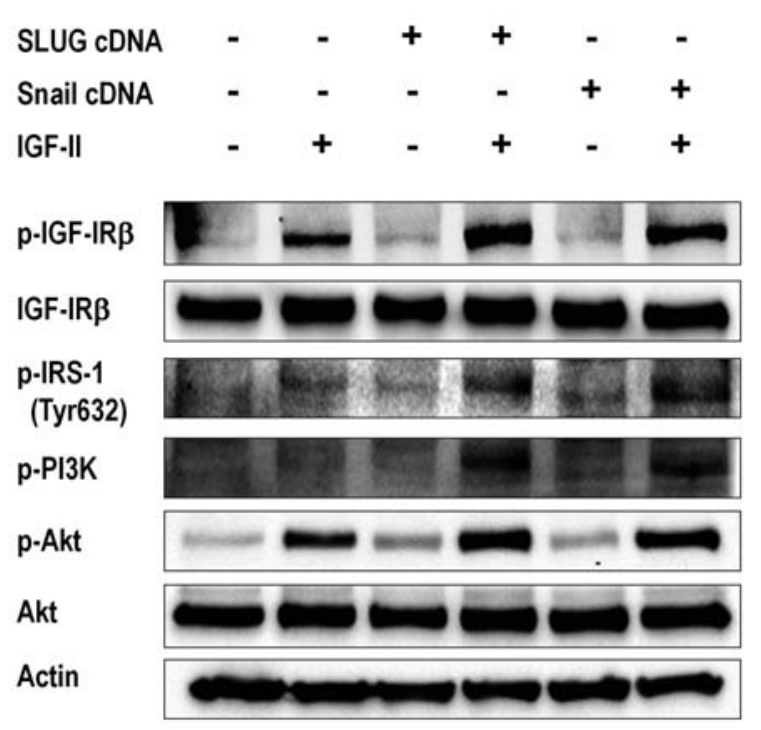

C

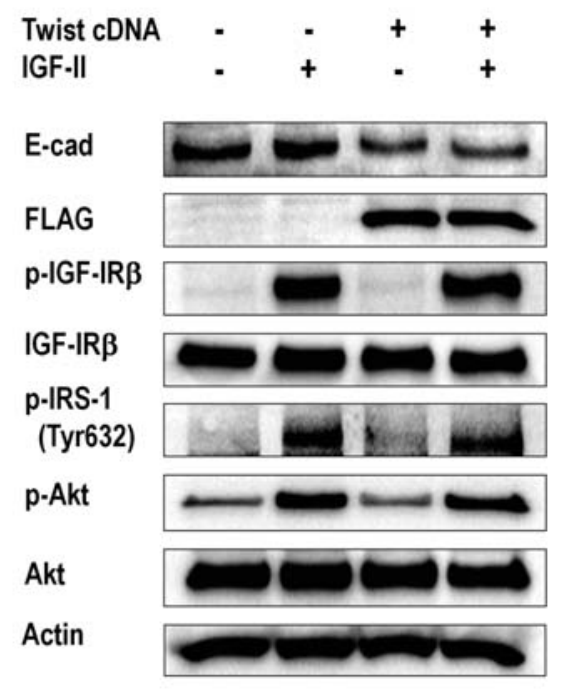

Figure 7. Transient cDNA transfection experiments. A, 60\%-confluent MDCK cells were transfected with 0.5 or $2 \mu \mathrm{g}$ cDNA of human Snail. At $24 \mathrm{~h}$ after the transfection, the cells were harvested and used in immunoblot analysis. Mock, empty vector. B and C, Enhanced sensitivity to IGF-II stimulation in MDCK cells transiently transfected with Snail or SLUG, but not with Twist cDNA $(2 \mu \mathrm{g})$. After $24 \mathrm{~h}$ of cDNA transfection, MDCK cells were cultured in serum-free medium for $12 \mathrm{~h}$ and then stimulated with $10 \mathrm{ng} / \mathrm{ml} \mathrm{IGF-II} \mathrm{for} 30 \mathrm{~min}$. zinc-finger-type Snail and SLUG. This finding, although preliminary, suggested a distinct transcriptional mechanism regulating the EMT-associated IGF-IR signaling.

Discovery of the dual Akt-activating pathways prompted us to investigate the apoptosis-inducing potential in targeting both IGF-IR and Src. This concept was inspired from recent progress in developing specific inhibitors of IGF-IR activity (35). If the Src inhibitor PP2 was combined with emerging IGF-IR inhibitors instead of with the fragile peptide IGFBP-3, more profound apoptosis of cancer cells might be expected. Secondary diabetes mellitus has been reported as an adverse effect of the IGF-IR inhibitors, although it could be controlled by metformin (35). In addition, novel Src inhibitors, including AZD0530, are emerging and are in clinical development (36). Thus, the dual inhibition of IGF-IR and Src would be beneficial in not only enhancing the therapeutic efficacy but also decreasing the severity of hyperglycemia.

\section{Acknowledgments}

This work was supported in part by a grant from a project for establishing new high technology research centers and in part by a grant from the 21st Century COE Program for Medical Science, from the Ministry of Education, Culture, Sports, Science, and Technology, of Japan. The authors thank Dr Ming Yue Jin, Dr Heiichi Hirano, and Ms. Masako Hayakawa for technical assistance.

\section{References}

1. Thiery JP and Sleeman JP: Complex networks orchestrate epithelial-mesenchymal transitions. Nat Rev Mol Cell Biol 7: 131-142, 2006.

2. Savagner P, Yamada KM and Thiery JP: The zinc-finger protein slug causes desmosome dissociation, an initial and necessary step for growth factor-induced epithelial-mesenchymal transition. J Cell Biol 137: 1403-1419, 1997.

3. Nieto MA: The snail superfamily of zinc-finger transcription factors. Nat Rev Mol Cell Biol 3: 155-166, 2002.

4. Barrallo-Gimeno A and Nieto MA: The Snail genes as inducers of cell movement and survival: implications in development and cancer. Development 132: 3151-3161, 2005.

5. Guarino M, Rubino B and Ballabio G: The role of epithelialmesenchymal transition in cancer pathology. Pathology 39: 305-318, 2007.

6. Larsson O, Girnita A and Girnita L: Role of insulin-like growth factor 1 receptor signalling in cancer. Br J Cancer 92: 2097-2101, 2005.

7. Irie HY, Pearline RV, Grueneberg D, et al: Distinct roles of Akt 1 and Akt 2 in regulating cell migration and epithelialmesenchymal transition. J Cell Biol 171: 1023-1034, 2005.

8. Kim HJ, Litzenburger BC, Cui X, et al: Constitutively active type I insulin-like growth factor receptor causes transformation and xenograft growth of immortalized mammary epithelial cells and is accompanied by an epithelial-to-mesenchymal transition mediated by NF-kappaB and snail. Mol Cell Biol 27: 3165-3175, 2007.

9. Gibson SL, Ma A and Shaw LM: Divergent roles for IRS-1 and IRS-2 in breast cancer metastasis. Cell Cycle 6: 631-637, 2007.

10. Guvakova MA and Surmacz E: The activated insulin-like growth factor I receptor induces depolarization in breast epithelial cells characterized by actin filament disassembly and tyrosine dephosphorylation of FAK, Cas, and paxillin. Exp Cell Res 251: 244-255, 1999. 
11. Sandhu MS, Dunger DB and Giovannucci EL: Insulin, insulinlike growth factor-I (IGF-I), IGF binding proteins, their biologic interactions, and colorectal cancer. J Natl Cancer Inst 94: 972-980, 2002.

12. Renehan AG, Zwahlen M, Minder C, O'Dwyer ST, Shalet SM and Egger M: Insulin-like growth factor (IGF)-I, IGF binding protein-3, and cancer risk: systematic review and meta-regression analysis. Lancet 363: 1346-1353, 2004.

13. Boyer B, Roche S, Denoyelle M and Thiery JP: Src and Ras are involved in separate pathways in epithelial cell scattering. EMBO J 16: 5904-5913, 1997.

14. Avizienyte E, Wyke AW, Jones RJ, et al: Src-induced deregulation of E-cadherin in colon cancer cells requires integrin signalling. Nat Cell Biol 4: 632-638, 2002.

15. Coluccia AM, Benati D, Dekhil H, De Filippo A, Lan C and Gambacorti-Passerini C: SKI-606 decreases growth and motility of colorectal cancer cells by preventing pp60(c-Src)-dependent tyrosine phosphorylation of beta-catenin and its nuclear signaling. Cancer Res 66: 2279-2286, 2006.

16. Nguyen KT, Wang WJ, Chan JL and Wang LH: Differential requirements of the MAP kinase and PI3 kinase signaling pathways in Src- versus insulin and IGF-1 receptors-induced growth and transformation of rat intestinal epithelial cells. Oncogene 19: 5385-5397, 2000.

17. Peterson JE, Kulik G, Jelinek T, Reuter CW, Shannon JA and Weber MJ: Src phosphorylates the insulin-like growth factor type I receptor on the autophosphorylation sites. Requirement for transformation by src. J Biol Chem 271: 31562-31571, 1996.

18. Valentinis B, Morrione A, Taylor SJ and Baserga R: Insulin-like growth factor I receptor signaling in transformation by src oncogenes. Mol Cell Biol 17: 3744-3754, 1997.

19. Sekharam M, Nasir A, Kaiser HE and Coppola D: Insulin-like growth factor 1 receptor activates c-SRC and modifies transformation and motility of colon cancer in vitro. Anticancer Res 23: $1517-1524,2003$.

20. Hakam A, Fang Q, Karl R and Coppola D: Coexpression of IGF-1R and c-Src proteins in human pancreatic ductal adenocarcinoma. Dig Dis Sci 48: 1972-1978, 2003.

21. Kobayashi N, Miyazaki M, Fukaya K, et al: Transplantation of highly differentiated immortalized human hepatocytes to treat acute liver failure. Transplantation 69: 202-207, 2000.

22. Shishido S, Koga H, Harada M, et al: Hydrogen peroxide overproduction in megamitochondria of troglitazone-treated human hepatocytes. Hepatology 37: 136-147, 2003.

23. Shiota M, Izumi H, Onitsuka T, et al: Twist promotes tumor cell growth through YB-1 expression. Cancer Res 68: 98-105, 2008 .
24. Maeyama M, Koga H, Selvendiran K, et al: Switching in discoid domain receptor expressions in SLUG-induced epithelialmesenchymal transition. Cancer (In press).

25. Guo Y, Zi X, Koontz Z, et al: Blocking Wnt/LRP5 signaling by a soluble receptor modulates the epithelial to mesenchymal transition and suppresses met and metalloproteinases in osteosarcoma Saos-2 cells. J Orthop Res 25: 964-971, 2007.

26. Peterson JE, Jelinek T, Kaleko M, Siddle K and Weber MJ: c phosphorylation and activation of the IGF-I receptor in srctransformed cells. J Biol Chem 269: 27315-27321, 1994.

27. Peinado H, Olmeda D and Cano A: Snail, Zeb and bHLH factors in tumour progression: an alliance against the epithelial phenotype? Nat Rev Cancer 7: 415-428, 2007.

28. Hynes NE and Lane HA: ERBB receptors and cancer: the complexity of targeted inhibitors. Nat Rev Cancer 5: 341-354, 2005.

29. Ando Y and Jensen PJ: Epidermal growth factor and insulin-like growth factor I enhance keratinocyte migration. J Invest Dermatol 100: 633-639, 1993.

30. Thomson S, Buck E, Petti F, et al: Epithelial to mesenchymal transition is a determinant of sensitivity of non-small-cell lung carcinoma cell lines and xenografts to epidermal growth factor receptor inhibition. Cancer Res 65: 9455-9462, 2005

31. Jechlinger M, Sommer A, Moriggl R, et al: Autocrine PDGFR signaling promotes mammary cancer metastasis. J Clin Invest 116: 1561-1570, 2006.

32. Del Castillo G, Murillo MM, Alvarez-Barrientos A, et al: Autocrine production of TGF-beta confers resistance to apoptosis after an epithelial-mesenchymal transition process in hepatocytes: role of EGF receptor ligands. Exp Cell Res 312: 2860-2871, 2006.

33. Sakai D, Suzuki T, Osumi N and Wakamatsu Y: Cooperative action of Sox9, Snail2 and PKA signaling in early neural crest development. Development 133: 1323-1333, 2006.

34. Sun $H$ and Baserga R: The role of insulin receptor substrate-1 in transformation by v-src. J Cell Physiol 215: 725-732, 2008.

35. Haluska P, Carboni JM, Loegering DA, et al: In vitro and in vivo antitumor effects of the dual insulin-like growth factor-I/insulin receptor inhibitor, BMS-554417. Cancer Res 66: 362-371, 2006.

36. Hiscox S, Jordan NJ, Smith C, et al: Dual targeting of Src and ER prevents acquired antihormone resistance in breast cancer cells. Breast Cancer Res Treat (In press). 